\title{
Excesso de peso em crianças e adolescentes sobreviventes de leucemia linfoide aguda - estudo de coorte
}

\section{Excessive weight in children and adolescents surviving acute lymphoid leukemia - a cohort study}

João Guilherme B. Alves ${ }^{1}$

Clarissa M. A. Pontes ${ }^{2}$

Mecneide M. Lins ${ }^{3}$
A leucemia linfoide aguda (LLA) é a neoplasia maligna mais comum na infância. A taxa de cura é hoje em torno de $80 \%$ e entre os sobreviventes tem sido descrito um aumento de risco de obesidade. Entretanto, não há estudos sobre esse risco em países em desenvolvimento, especialmente naqueles que atravessam um momento de transição nutricional, como o Brasil. O objetivo do estudo foi verificar a frequência de excesso de peso em crianças e adolescentes sobreviventes de leucemia linfoide aguda. Foi realizado um estudo de coorte retrospectivo com 101 crianças e 19 adolescentes sobreviventes de leucemia linfoide aguda. Todos esses pacientes foram tratados no Instituto de Medicina Integral Prof. Fernando Figueira (IMIP), entre os anos de 1998 e 2002. O seguimento foi de quatro anos e meio, sendo o índice de massa corpórea (IMC) calculado no momento do diagnóstico, ao final de tratamento e dois anos após o término da terapia. A idade média do diagnóstico foi de 4,6 (2,1) anos nas crianças e de 12,2 (1,9) anos nos adolescentes. O tempo de tratamento foi de 2,6 (1,0) anos. O IMC aumentou de 16,1 $(2,3)$ para $19,1(3,5)$ após o tratamento; $p<0.001$. A frequência de excesso de peso passou de 17/120 (14,2\%) para 55/120 (45,8\%) após o tratamento; $p<0,001$. Não houve variações significativas da frequência de excesso de peso e do IMC, entre o término do tratamento e dois anos após: 55/120 (45,8\%) vs 46/120 (38,3\%), $p=$ 0,295, e IMC de 19,1 (3,5) vs 19,4 (3,5); $p=0,178$. O tratamento da leucemia linfoide aguda aumenta o risco de excesso de peso entre as crianças e adolescentes sobreviventes. Rev. Bras. Hematol. Hemoter.

Palavras-chave: Leucemia; sobrepeso; obesidade; sobreviventes.

\section{Introdução}

A leucemia linfoide aguda (LLA), neoplasia maligna mais comum na faixa etária pediátrica, é atualmente curável em cerca de $80 \%$ dos pacientes. ${ }^{1,2}$ Isso tem resultado em uma crescente população de sobreviventes e gerado vários questionamentos sobre um maior risco de outras afecções nesse grupo populacional. ${ }^{3,4}$

A obesidade, um dos principais problemas de saúde pública no mundo no século atual, tanto nos países desen- volvidos como nos em desenvolvimento, tem sido apontada como um dos efeitos tardios da terapêutica nos sobreviventes de leucemia LLA. ${ }^{5-10} \mathrm{~A}$ obesidade representa um importante fator de risco para as doenças cardiovasculares, principais causas de morte no mundo, além de frequentemente estar associada com comorbidades como diabetes tipo 2 , dislipidemias, distúrbios respiratórias, afecções osteoarticulares, entre outras, inclusive na infância. ${ }^{11,12,13}$

Alguns estudos apontam que o maior risco de obesidade em sobreviventes de LLA seria pela deficiência

\footnotetext{
${ }^{I}$ Médico. Coordenador do Programa de Pós-Graduação do Instituto de Medicina Integral Prof. Fernando Figueira (IMIP) - Recife-PE. ${ }_{3}^{2}$ Aluno do Curso de Medicina - Escola Pernambucana de Medicina - Recife-PE.

${ }^{3}$ Médica do Serviço de Oncologia Infantil do Instituto de Medicina Integral Prof. Fernando Figueira (IMIP) - Recife-PE.
}

Instituto de Medicina Integral Prof Fernando Figueira (IMIP) - Recife-PE.

Correspondência: João Guilherme Bezerra Alves

Rua dos Coelhos, 300 - Boa Vista

50070-550 - Recife-PE - Brasil

E-mail: joaoguilherme@imip.org.br

Doi: 
do hormônio do crescimento na vida adulta..$^{14,15,16}$ Outra hipótese é a de que a irradiação craniana acarrete danos nos centros cerebrais que controlam o apetite, o comportamento alimentar e a composição corporal. ${ }^{17}$ Especula-se ainda que $o$ mecanismo de obesidade nesses pacientes seja multifatorial, podendo incluir anormalidades do balanço energético, diminuição da atividade física, alterações hormonais e uso de corticoides. ${ }^{18,19}$

Os efeitos do tratamento da LLA na composição corporal não têm sido estudados nos países em desenvolvimento, que vêm apresentando um maior crescimento da obesidade na população de baixa renda, como o Brasil, que atravessa um rápido momento de transição nutricional. Dessa forma, este estudo tem como objetivo verificar a frequência de obesidade em sobreviventes que realizaram o tratamento de LLA na infância ou adolescência.

\section{Casuística e Método}

Realizou-se um estudo de coorte retrospectivo com 120 indivíduos de 2 a 17 anos, sendo 101 crianças ( 5 a 9 anos) e 19 adolescentes ( 10 a 19 anos), 65 meninos e 55 meninas, todos diagnosticados, tratados e curados de LLA entre os anos de 1998 a 2002, no serviço de Oncologia do Instituto de Medicina Integral Prof. Fernando Figueira (IMIP). O IMIP é o maior hospital pediátrico do Norte-Nordeste do Brasil e o seu serviço de Oncologia atende uma média de 200 casos novos por ano de crianças e adolescentes com câncer, dos quais cerca de $40 \%$ com diagnóstico de leucemia.

Todos os participantes foram tratados com o protocolo XIIIB do Saint Judes Research Hospital, ${ }^{20}$ com o qual o IMIP mantém convênio de cooperação técnico-científica, e vem usando esse protocolo desde o ano de 1997. Esse protocolo é composto de uma fase de indução e consolidação com uma duração de oito semanas, seguido por uma manutenção com duração de 120 semanas e uma reindução nas semanas 16-21. A terapia direcionada ao SNC é realizada com elevadas doses de MTX associada a medicações intratecais. A radioterapia do crânio é reservada para os grupos de alto risco. As crianças foram classificadas em dois grupos: Risco Standard (idade entre $1 \mathrm{e}$ 10 anos, leucometria inicial $<25 \times 10 \mathrm{E} 9 / \mathrm{L}$, ausência de paralisia de nervos cranianos e LCR normal) e Alto Risco (demais pacientes que apresentem uma ou mais das características citadas, e que não apresentem uma boa resposta terapêutica com mais de 5\% de blastos na medula). Pacientes de alto risco foram submetidos à radioterapia de crânio com 1,5 Gy, quando não havia comprometimento do SNC e a leucometria se encontrava abaixo de $100.000 \mathrm{p} / \mathrm{mm}^{3}$, ou com 1,8 Gy, quando havia comprometimento do SNC ou a leucometria era maior ou igual a $100.000 \mathrm{p} / \mathrm{mm}^{3}$.
Os dados antropométricos foram coletados através dos prontuários dos pacientes, no momento do diagnóstico, no final do tratamento e dois anos após. O Índice de Massa Corporal (IMC) foi calculado pela divisão do peso $(\mathrm{kg})$ pela altura $(\mathrm{cm})$ elevada ao quadrado. Foram considerados como portadores de sobrepeso aqueles indivíduos que apresentavam um IMC entre os porcentuais 85 e 95 , e obesidade quando o porcentual estava acima de 95 . Foram utilizados como referência os dados do National Center to Health Statistics. ${ }^{21}$

O estudo recebeu aprovação prévia do Comitê de Ética em Pesquisa em Seres Humanos do IMIP, atendendo à Resolução n 196/96 do Conselho Nacional de Saúde.

Foi empregado o teste exato de Fisher na comparação das taxas de obesidade nos momentos distintos da evolução dos pacientes, sendo aceito um nível de significância de $\mathrm{p}<0.05$.

\section{Resultados}

Dos 120 sobreviventes estudados, 65 (54,2\%) eram do sexo masculino. A idade da confirmação diagnóstica de LLA variou de 2 a 17 anos; 4,6 anos ( $\mathrm{DP}=2,1)$ nas crianças e 12,2 anos $(\mathrm{DP}=1,9)$ nos adolescentes. $\mathrm{O}$ tempo médio de tratamento foi de 2,6 anos; $\mathrm{DP}=1,0$.

No momento da confirmação diagnóstica, 17 (14,2\%) dos 120 sobreviventes apresentavam excesso de peso. Esse número aumentou para $55(45,8 \%)$ ao término do tratamento. Entre o término do tratamento e dois anos após, não foram observadas modificações nas frequências de sobrepeso e obesidade (Tabela). A média do IMC variou de 16,1 (DP=2,3) no momento do diagnóstico de LLA para 19,1 $(\mathrm{DP}=3,5) ; \mathrm{p}<$ 0.001. Quando comparado o IMC do final do tratamento com o de dois anos após $(19,4 ; \mathrm{DP}=3,5)$, não houve diferença significativa $(p=0,18)$.

\begin{tabular}{|c|c|c|c|c|}
\hline $\begin{array}{l}\text { Sobrepeso/ } \\
\text { Obesidade }\end{array}$ & $\begin{array}{l}\text { Diagnóstico de } \\
\text { leucemia linfoide } \\
\text { aguda }\end{array}$ & $\begin{array}{l}\text { Término do } \\
\text { tratamento }\end{array}$ & $\begin{array}{l}\text { Dois anos após o } \\
\text { tratamento }\end{array}$ & $p$ \\
\hline Não & $103(85,8 \%)$ & $65(54,2 \%)$ & $74(61,7 \%)$ & $<0,001^{*}$ \\
\hline Sim & $17(14,2 \%)$ & $55(45,8 \%)$ & $46(38,3 \%)$ & 0,29 ** \\
\hline Total & $120(100,0 \%)$ & $120(100,0 \%)$ & $120(100,0 \%)$ & \\
\hline
\end{tabular}

* Diagnóstico e término do tratamento

** Final do tratamento e dois anos após

\section{Discussão}

Nossos achados indicaram que a frequência de excesso de peso quase triplicou (14,2\% versus $38,3 \%$ ) nas crianças e adolescentes, quando comparados o momento do diagnóstico com dois anos após o término do tratamento e cura da LLA. Esses valores observados de soprepeso e obesidade são 
mais elevados do que os descritos em populações dessa mesma faixa etária e região. ${ }^{22}$ Os estudos feitos nos países desenvolvidos, investigando adultos jovens, tratados na infância e adolescência, tendem a demonstrar uma maior frequência de excesso de peso entre os sobreviventes de LLA. ${ }^{23-26}$ Entretanto, não observamos, nesses estudos, aumentos de excesso de peso tão significativos como o detectado no presente trabalho. Algumas razões podem justificar essas diferenças. Inicialmente, em nossa região, Nordeste do Brasil, o diagnóstico de câncer na infância é realizado de forma mais tardia quando comparado com países desenvolvidos, ${ }^{27,28}$ devido, habitualmente, à demora, pelos pais, pela procura na assistência médica. Esse fato implica agravamento do estado nutricional da criança no momento do diagnóstico. Em segundo lugar, diferentemente dos países desenvolvidos, o Brasil, especialmente na região nordeste, ainda apresenta taxas significativas de baixo peso ao nascer, fator de risco para obesidade, desde que superados os agravos que levaram à subnutrição fetal. ${ }^{29}$ Registre-se ainda que maiores cuidados dietéticos são dispensados a crianças e adolescentes diagnosticados com LLA que, inclusive, muitas vezes, perduram após o tratamento. ${ }^{30,31}$ Esses fatos, corroborado pela clientela estudada ser reconhecidamente de baixa renda, o que determina uma maior procura por alimentos ricos em açúcar e gorduras, devido ao seu menor custo, podem justificar esses achados.

Por outro lado, os estudos aos quais comparamos nossos resultados foram todos realizados com adultos sobreviventes de LLA e não crianças ou adolescentes. É bem estabelecido que o risco de excesso de peso apresenta uma forte correlação positiva com a idade. ${ }^{33}$

Os estudos em relação aos fatores que, potencialmente, determinam o excesso de peso entre os sobreviventes de LLA, têm pesquisado eminentemente os fatores endógenos. Entre esses, a irradiação do SNC tem mostrado resultados discordantes. ${ }^{17,27,28}$ Para alguns autores, a presença da obesidade no momento do diagnóstico seria o principal fator determinante do excesso de peso entre os sobreviventes..$^{26}$ Em relação à leptina, hormônio produzido pelo tecido adiposo e que está envolvido no controle da saciedade, vários estudos têm demonstrado níveis mais elevados entre os sobreviventes da LLA, inclusive entre aqueles sem excesso de peso, o que fortalece o papel interativo dos fatores endógenos com os ambientais no desenvolvimento da obesidade após a cura da LLA. ${ }^{31}$

Registre-se que a casuística estudada era proveniente de um hospital público, que atende exclusivamente a pacientes do Sistema Único de Saúde (SUS), população reconhecidamente de baixa renda. Mesmo assim, tivemos porcentuais de excesso de peso na amostra estudada bem acima dos descritos em populações locais semelhantes. ${ }^{22} \mathrm{O}$ Brasil atravessa atualmente um movimento de transição nutricional e o excesso de peso vem apresentando um maior crescimento na população de baixa renda, tendo sido apon- tados como os principais determinantes dessa transição epidemiológica a inatividade física e o uso de dietas hipercalóricas, fatores mais observados nessas camadas com menor nível aquisitivo. ${ }^{34}$

É importante que sejam registradas algumas limitações de nosso estudo. Inicialmente, por tratar-se de um desenho retrospectivo, não obtivemos informações sobre o histórico familiar desses sobreviventes para excesso de peso, o que é reconhecidamente um fator de risco para a obesidade. Também não mensuramos a circunferência abdominal, medida considerada mais sensível e específica para o risco metabólico de indivíduos com excesso de peso. Nosso tempo de seguimento, apenas dois anos, não permitiu definir a manutenção do excesso de peso obtido após o tratamento da LLA, durante a vida adulta.

Nosso estudo, entretanto, além de ter sido o primeiro realizado em um país em desenvolvimento com o objetivo de verificar a presença de excesso de peso em sobreviventes de LLA, contou com um número adequado de participantes. A pesquisa foi realizada no maior centro de oncologia infantil do Nordeste, IMIP, que recebe um forte suporte do Saint Judes Children's Hospital, mantendo os mesmos protocolos de tratamento e seguimento dos pacientes, além de reuniões semanais através da telemedicina com o corpo clínico.

Concluímos que o tratamento da LLA em crianças e adolescentes acarreta um aumento significativo de excesso de peso ente os sobreviventes. Esse efeito parece ser mais intenso do que nos países desenvolvidos, o que reforça o papel dos fatores ambientais no desencadeamento da obesidade em sobreviventes de LLA. Novos estudos de coorte, com um maior tempo de observação, necessitam ser realizados em países em desenvolvimento para um melhor esclarecimento sobre a obesidade e comorbidades em sobreviventes de LLA.

\footnotetext{
Abstract

Acute lymphoblastic leukemia (ALL) is the most common malignancy of childhood. The cure rate is now about $80 \%$ but an increased risk for obesity has been described among survivors. Even so there are no studies reporting this risk in developing countries especially in countries in which the problem of dietary deficiency is rapidly shifting to one of dietary excess. The purpose of this study was to assess the frequency of excessive weight in child and teenage survivors of ALL. A retrospective cohort study was carried out of 101 children and 19 adolescents diagnosed and treated for ALL between 1998 and 2002 in the Instituto de Medicina Integral Prof. Fernando Figueira (IMIP). The body mass index (BMI) was calculated at the time of diagnosis, at the end of therapy and two years later. The mean age at diagnosis was $4.6( \pm 2.1)$ years in children and 12.2 $( \pm 1.9)$ years in adolescents. The duration of treatment was $2.6( \pm 1.0)$ years. The BMI changed from 16.1 ( \pm 2.3$)$ to 19.1 ( \pm 3.5$)$ after treatment; $p<0.001$. Among the survivors $17 / 120$ (14.2\%) were overweight at the time of diagnosis and 55/120 (45.8\%) after treatment $(p<0.001)$. There were no significant differences between the percentage of overweight individuals and BMI between the end
} 
of treatment and two years later: $55 / 120(45.8 \%)$ vs. 46/120(38.3\%) $(p=0.295)$ and BMI $19.1( \pm 3.5)$ vs. $19.4( \pm 3.5)(p=0.178)$. Treatment of acute lymphoblastic leukemia increases the risk of excessive weight in child and adolescent survivors. Rev. Bras. Hematol. Hemoter.

Key words: Leukemia; overweight; obesity; survivors.

\section{Referências Bibliográficas}

1. Pui CH, Robison LL, Look AT. Acute lymphoblastic leukaemia. Lancet. 2008;371(9617):1030-43.

2. Armstrong GT, Sklar CA, Hudson MM, Robison LL. Long-term health status among survivors of childhood cancer: does sex matter? J Clin Oncol. 2007;25(28):4477-89.

3. Desandes E. Survival from adolescent cancer. Cancer Treat Rev. 2007;33(7):609-15.

4. Robison LL, Bhatia S. Late-effects among survivors of leukaemia and lymphoma during childhood and adolescence. Br J Haematol. 2003;122(3):345-59

5. Odame I, Reilly JJ, Gibson BE, Donaldson MD. Patterns of obesity in boys and girls after treatment for acute lymphoblastic leukaemia. Arch Dis Child. 1994;71(2):147-9.

6. Davies HA, Didcock E, Didi M, Ogilvy-Stuart A, Wales JK, Shalet SM. Growth, puberty and obesity after treatment for leukaemia. Acta Paediatr Suppl. 1995;411:45-50.

7. Didi M, Didcock E, Davies HA, Ogilvy-Stuart AL, Wales JK, Shalet SM. High incidence of obesity in young adults after treatment of acute lymphoblastic leukemia in childhood. J Pediatr. 1995;127 (1):63-7.

8. Pagano L, De Rosa G, Voso MT, Marra R, Testa A, Leone G. Prevalence of obesity in young adults with acute lymphoblastic leukemia. Int J Clin Lab Res. 1994;24(2):117-9.

9. Van Dongen-Melman JE, Hokken-Koelega AC, Hählen K, De Groot A, Tromp CG, Egeler RM. Obesity after successful treatment of acute lymphoblastic leukemia in childhood. Pediatr Res. 1995; 38(1):86-90.

10. Oeffinger KC, Mertens AC, Sklar CA, Yasui Y, Fears T, Stovall M, et al. Obesity in adult survivors of childhood acute lymphoblastic leukemia: a report from the Childhood Cancer Survivor Study. J Clin Oncol. 2003;21(7):1359-65.

11. Pérez Pérez A, Ybarra Muñoz J, Blay Cortés V, de Pablos Velasco P. Obesity and cardiovascular disease. Public Health Nutr. 2007; 10(10A):1156-63.

12. Behn A, Ur E. The obesity epidemic and its cardiovascular consequences. Curr Opin Cardiol. 2006;21(4):353-60.

13. Choudhary AK, Donnelly LF, Racadio JM, Strife JL. Diseases associated with childhood obesity. AJR Am J Roentgenol. 2007; 188(4):1118-30.

14. Warner JT, Gregory JW, Webb DK. Patterns of obesity in boys and girls after treatment for acute lymphoblastic leukaemia. Arch Dis Child. 1995;72(1):97.

15. Zee P, Chen $\mathrm{CH}$. Prevalence of obesity in children after therapy for acute lymphoblastic leukemia. Am J Pediatr Hematol Oncol. 1986;8(4):294-9.

16. Sainsbury CP, Newcombe RG, Hughes IA. Weight gain and height velocity during prolonged first remission from acute lymphoblastic leukaemia. Arch Dis Child. 1985;60(9):832-6.

17. Jarfelt M, Bjarnason R, Lannering B. Young adult survivors of childhood acute lymphoblastic leukemia: spontaneous GH secretion in relation to CNS radiation. Pediatr Blood Cancer. 2004;42(7): 582-8.
18. Warner JT, Evans WD, Webb DK, Gregory JW. Body composition of long-term survivors of acute lymphoblastic leukaemia. Med Pediatr Oncol. 2002;38(3):165-72.

19. Jarfelt M, Lannering B, Bosaeus I, Johannsson G, Bjarnason R. Body composition in young adult survivors of childhood acute lymphoblastic leukaemia. Eur J Endocrinol. 2005;153(1):81-9.

20. Pui CH, Sandlund JT, Pei D, Campana D, Rivera GK, Ribeiro RC, et al. Improved outcome for children with acute lymphoblastic leukemia: results of Total Therapy Study XIIIB at St Jude Children's Research Hospital. Blood. 2004;104(9):2690-6.

21. National Center to Health Statistics. Clinical Growth Charts. Acessado em 14/09/07 no endereço: http://www.cdc.gov/nchs/ about/major/nhanes/growthcharts/clinical_charts.htm

22. Batista Filho M, Rissin A. Nutritional transition in Brazil: geographic and temporal trends. Cad Saude Publica. 2003;19 Suppl 1:S181-91.

23. Rogers PC, Meacham LR, Oeffinger KC, Henry DW, Lange BJ. Obesity in pediatric oncology. Pediatr Blood Cancer. 2005; 45 (7):881-91.

24. Oeffinger KC. Are survivors of acute lymphoblastic leukemia (ALL) at increased risk of cardiovascular disease? Pediatr Blood Cancer. 2008;50(2 Supp1):462-7.

25. Asner S, Ammann RA, Ozsahin H, Beck-Popovic M, von der Weid NX. Obesity in long-term survivors of childhood acute lymphoblastic leukemia. Pediatr Blood Cancer. 2008;51(1):118-22.

26. Razzouk BI, Rose SR, Hongeng S, Wallace D, Smeltzer MP, Zacher $\mathrm{M}$, et al. Obesity in survivors of childhood acute lymphoblastic leukemia and lymphoma. J Clin Oncol. 2007;25(10):1183-9.

27. Viana MB, Fernandes RA, de Carvalho RI, Murao M. Low socioeconomic status is a strong independent predictor of relapse in childhood acute lymphoblastic leukemia. Int J Cancer Suppl. 1998;11:56-61.

28. Hsu SC, Metzger ML, Hudson MM, Pedrosa F, Lins M, Pedrosa M, et al. Comparison of treatment outcomes of childhood Hodgkin lymphoma in two US centers and a center in Recife, Brazil. Pediatr Blood Cancer. 2007;49(2):139-44.

29. Moor V, Davies M. Early life influences on later health: the role of nutrition. Asia Pac J Clin Nutr. 2001;10(2):113-7.

30. Siviero-Miachon AA, Spinola-Castro AM, Tosta-Hernandez PD, de Martino Lee ML, Petrilli AS. Leptin assessment in acute lymphocytic leukemia survivors: role of cranial radiotherapy? J Pediatr Hematol Oncol. 2007;29(11):776-82.

31. Garmey EG, Liu Q, Sklar CA, Meacham LR, Mertens AC, Stovall MA, et al. Longitudinal changes in obesity and body mass index among adult survivors of childhood acute lymphoblastic leukemia: a report from the Childhood Cancer Survivor Study. J Clin Oncol. 2008;26(28):4639-45.

32. Papadia C, Naves LA, Costa SS, Vaz JA, Domingues L, Casulari LA. Incidence of obesity does not appear to be increased after treatment of acute lymphoblastic leukemia in Brazilian children: role of leptin, insulin, and IGF-1. Horm Res. 2007;68(4):164-70.

33. Kaila B, Raman M. Obesity: a review of pathogenesis and management strategies. Can J Gastroenterol. 2008;22(1):61-8.

34. Monteiro CA, Conde WL, Popkin BM. The burden of disease from undernutrition and overnutrition in countries undergoing rapid nutrition transition: a view from Brazil. Am J Public Health. 2004; 94(3):433-4.

Avaliação: Editor e dois revisores externos

Conflito de interesse: sem conflito de interesse

Recebido: 27/01/2009

Aceito após modificações: 11/06/2009 